\title{
Costa Rica sin Fronteras
}

Pensar en Movimiento:

Revista de Ciencias del Ejercicio y la Salud

ISSN 1659-4436

Vol. 10, No.2, pp. 1-3

\section{COSTA RICA SIN FRONTERAS \#6}

En esta sección se publican los resúmenes en español de artículos que han sido publicados por investigadoras/es de universidades costarricenses en otras revistas en el mundo, con su debida referencia al trabajo original, y con una breve explicación de dónde se realizó la investigación. Los artículos originales han sido publicados en otros idiomas; las revistas tienen consejo editorial y manejan un proceso de revisión por pares.

Los resúmenes corresponden a estudios que se conforman a los criterios generales de la revista, esto es, se trata de "... estudios experimentales o que hagan recomendaciones concretas para solucionar problemas o preguntas relevantes (...) trabajos originales o de meta-análisis." Solicitamos a las personas que han publicado este tipo de trabajos en otros idiomas que no los hagan saber, para incluir sus resúmenes aquí. 


\section{CAMBIOS EN EL PESO CORPORAL EN ATLETAS INFANTILES Y JUVENILES DURANTE UNA COMPETENCIA DE TRIATLÓN}

Aragón Vargas, L. F., Wilk, B., Timmons, B. W. \& Bar-Or, O. (2012). Body Weight Changes in Child and Adolescent Athletes during a Triathlon Competition. European Journal of Applied Physiology. doi: http://dx.doi.org/10.1007/s00421-012-2431-8.

Examinamos atletas jóvenes durante un triatlón realizado en clima caliente. Obtuvimos datos completos o parciales de 95 atletas que compitieron en el Campeonato Nacional de Triatlón en Costa Rica. La edad y peso (PC) de los participantes fueron $13.1 \pm 2.5$ años y $46.3 \pm$ $11.5 \mathrm{~kg}$ (Media $\pm \mathrm{DE}$ ), respectivamente. Los requisitos de la competencia fueron: $500 \mathrm{~m}$ de natación, $15 \mathrm{~km}$ de ciclismo y $3.5 \mathrm{~km}$ de carrera para los jóvenes (9-13 años); $800 \mathrm{~m}$ de natación, $30 \mathrm{~km}$ de ciclismo y $8 \mathrm{~km}$ de carrera para los mayores (14-17 años). El índice WBGT el día de la competencia fue $>31^{\circ} \mathrm{C}$. Se obtuvieron registros del PC pre- y post- competencia para 92 atletas, de los cuales se recolectaron datos de rendimiento de 83. Se obtuvo además información acerca de los síntomas experimentados durante la carrera de 95 atletas. Se calculó el cambio del peso corporal ( $\% \Delta \mathrm{PC})$, el cual estuvo entre $+0.6 \%$ y $-2.4 \%$ para los varones jóvenes $(-1.2 \pm 0.9 \%)$, entre $+0.7 \%$ y $-2.5 \%$ para las chicas jóvenes $(-1.3 \pm 0.9 \%)$, entre $0 \%$ y $-2.8 \%$ para las chicas mayores $(-1.3 \pm 0.9 \%)$, y entre $+0.6 \%$ y $-4.5 \%$ para los varones mayores $(-1.7 \pm 1.1 \%)$. Dieciocho participantes no reportaron síntoma médico alguno. De los 77 participantes que reportaron síntomas, 42.9\% reportaron agotamiento/fatiga, $36.4 \%$ reportaron cólico/calambres, y $23.4 \%$ reportaron mareos. Aquellos participantes que no reportaron síntoma médico alguno alcanzaron un $\% \Delta \mathrm{PC}$ casi idéntico $(p=0.99)$ al de aquellos que reportaron al menos un síntoma. El \% $\triangle P C$ fue más negativo $(p=$ $0.005)$ en los participantes que reportaron mareos $(-1.9 \% \Delta \mathrm{PC})$ comparado con quienes no los reportaron $(-1.4 \% \triangle \mathrm{PC})$. El \% $\triangle \mathrm{PC}$ estuvo correlacionado con el rendimiento en las chicas jóvenes $(r=0.47, p=0.02)$ y los varones mayores $(r=0.51, p=0.01)$, mostrando una tendencia en los varones jóvenes $(r=0.41, p=0.053)$ pero no en las chicas mayores $(r=$ $0.004, p=0.99$ ). Los y las atletas jóvenes que participan en un triatlón en clima caliente pueden tolerar la deshidratación desde leve hasta moderada, sin efectos negativos sobre su propia valoración de la salud.

PALABRAS CLAVES: Estrés térmico, termorregulación, hidratación

Publicado Online First en Junio de 2012. Este estudio de campo se realizó en Playas del Coco, Guanacaste (Costa Rica) durante el Triatlón de El Coco. Fue un esfuerzo conjunto entre la Universidad de McMaster en Ontario, Canadá y la Universidad de Costa Rica. Recordamos con admiración y respeto al Dr. Oded Bar-Or, gestor de la iniciativa, q.d.g.D. Colaborador: Luis Fernando Aragón Vargas.

La revista European Journal of Applied Physiology reporta un factor de impacto de 2.147 (año 2011). 


\section{EJERCICIO DE PILATES PARA EL DOLOR LUMBAR PERSISTENTE NO ESPECÍFICO Y LA DISCAPACIDAD FUNCIONAL ASOCIADA: UN METAANÁLISIS CON METAREGRESIÓN}

Aladro-Gonzalvo, A. R., Araya-Vargas, G.A., Machado-Díaz, M. \& Salazar-Rojas, W. (2012). Pilates-based exercise for persistent, non-specific low back pain and associated functional disability: A meta-analysis with meta-regression. Journal of Bodywork and Movement Therapies. doi: http://dx.doi.org/10.1016/j.jbmt.2012.08.003

Objetivo. El objetivo de este estudio fue revisar sistemáticamente y aplicar análisis de regresión a los ensayos controlados aleatorizados (ECAs) que evaluaron la efectividad del ejercicio Pilates como recurso para mejorar el dolor lumbar persistente no específico y la discapacidad funcional. Metodología. Se realizó una búsqueda electrónica en bases de datos desde enero de 1950 hasta marzo de 2011. Los artículos fueron elegibles para su inclusión si estos fueron ECAs que compararon el ejercicio Pilates con un tratamiento placebo (TP), intervenciones mínimas (IM) $u$ otros tratamientos de fisioterapia (OTF). Resultados. Se incluyeron nueve ensayos. Pilates fue moderadamente superior a OTF en la reducción de la discapacidad ([TE] agrupado ponderado $=-0.55$, IC 95\% $[-0.08,-1.03]$, pero no hubo diferencia entre OTF y Pilates en el alivio del dolor. Cuando se le comparó con intervenciones mínimas, el ejercicio Pilates proporcionó un alivio del dolor entre moderado y superior (TE agrupado ponderado $=-0.44$, IC 95\% $[-0.09,-0.80]$, y una reducción similar de la discapacidad. El modelo estadístico utilizado no detectó variable predictora alguna. Conclusiones. Debido a la presencia de cointervenciones y a la débil calidad de algunos de los estudios, estos resultados deben interpretarse con cautela.

PALABRAS CLAVES: Pilates, Revisión sistemática, Ejercicio terapéutico, Terapia complementaria, Dolor lumbar, Rehabilitación, Metaregresión.

Publicado en línea en setiembre de 2012. Esta es una publicación de un trabajo de metaanálisis de Arián Aladro Gonzalvo, M.Sc., realizado cuando era estudiante del programa de Maestría Académica en Ciencias del Movimiento Humano de la Escuela de Educación Física y Deportes de la Universidad de Costa Rica. Colaborador: Arián R. AladroGonzalvo.

La revista Journal of Bodywork and Movement Therapies no reporta factor de impacto para el año 2012. Está indexada en Medline.

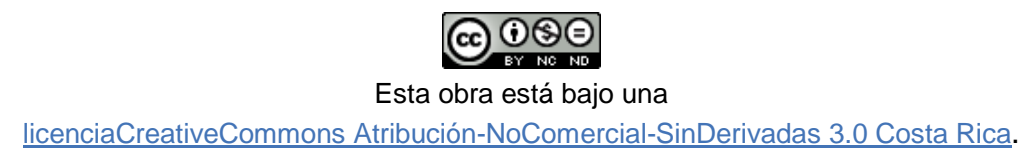

